\title{
Diagnostic yield of transbronchial needle aspiration for lymphoma
}

\author{
B Sonnekus, ${ }^{1} \mathrm{MB}$ ChB, MMed, FCP; J Steenkamp, ${ }^{2} \mathrm{MB}$ ChB, FCPath; M Louw, ${ }^{3} \mathrm{MB}$ ChB, MMed; \\ C F N Koegelenberg, ${ }^{4} \mathrm{MB}$ ChB, MMed, FCP, MRCP, Cert Pulmonology, PhD \\ ${ }^{1}$ Department of Medicine, Stellenbosch University and Tygerberg Hospital, Cape Town, South Africa \\ ${ }^{2}$ Division of Haemato-Pathology, Department of Pathology and National Health Laboratory Service, Stellenbosch University and Tygerberg Hospital, Cape Town, \\ South Africa \\ ${ }^{3}$ Division of Anatomical Pathology, Department of Pathology and National Health Laboratory Service, Stellenbosch University and Tygerberg Hospital, Cape \\ Town, South Africa \\ ${ }^{4}$ Division of Pulmonology, Department of Medicine, Stellenbosch University and Tygerberg Hospital, Cape Town, South Africa
}

Corresponding author: B Sonnekus (brentiasonnekus@yahoo.com)

Background. Transbronchial needle aspiration (TBNA) is a minimally invasive bronchoscopic technique that is cost-effective and safe for diagnosing mediastinal and hilar adenopathy in lung cancer, other malignancies, sarcoidosis and infectious processes such as tuberculosis. Few studies have analysed the sensitivity, specificity and predictive values of TBNA for diagnosing lymphoma.

Objective. To evaluate the diagnostic yield of TBNA for diagnosing mediastinal and hilar adenopathy in suspected lymphoma.

Methods. We performed a retrospective analysis of collected data of patients with mediastinal and hilar adenopathy adjacent to the tracheobronchial tree detected by thoracic computed tomography, who underwent TBNA at Tygerberg Hospital between July 2010 and June 2013. We included 25 patients with suspected or proven lymphoma. Histology was used as the gold standard.

Results. Adequate samples for cytological evaluation were obtained for 22 (88\%) patients. Cytological diagnosis was possible for 8 (32\%). For 17 (68\%) who could not be diagnosed by TBNA alone, histology provided final diagnosis. Rapid on-site examination (ROSE) was performed in 23 (92\%). In 17/23 (74\%) cases, these had similar results to formal cytology. Only 4 (16\%) had flow cytometry requested. Twelve (48\%) had lymphoma confirmed on histology. TBNA cytology had $100 \%$ specificity and positive predictive value for suspicion of lymphoma. Sensitivity was $33 \%$ and negative predictive value $62 \%$.

Conclusion. TBNA is an appropriate first-line diagnostic procedure in evaluating mediastinal and hilar lymphadenopathy in suspected lymphoma. Biopsy should be the immediate second-line procedure when ROSE/cytology is suspicious of lymphoma or shows atypical cells. Patients with negative TBNA cytology, but high clinical or radiological suspicion of lymphoma, should be further investigated.

S Afr Respir J 2016;22(3):67-72. DOI:10.7196/SARJ.2016.v22i3.80

Lymphoma is one of the top 10 most common cancers according to the Cancer Association of South Africa (CANSA) (last updated info, 2010). ${ }^{[1]}$ The delayed diagnosis or misdiagnosis of lymphoma is an important clinical problem in SA. Owing to the varied clinical picture, especially in HIV-positive patients, symptoms may mimic other diseases, particularly tuberculosis (TB). The 2011 World Health Organization (WHO) report for SA revealed a treatment failure rate of $27 \%$ for smear-positive TB and $36 \%$ for smear-negative/extrapulmonary TB. ${ }^{[2]}$ WHO and SA guidelines suggest empirical treatment of TB, particularly in HIVpositive patients. ${ }^{[3,4]}$ However, these guidelines stress the importance of sample culture testing, with follow-up investigations and reviews of treatment response. TB responds rapidly in HIV-positive patients. ${ }^{[5]}$ Lymphoma must be considered an important differential when TB cannot be confirmed in patients presenting with lymphadenopathy with/without constitutional symptoms.

In a retrospective study, conducted in rural KwaZulu-Natal, Puvaneswaran et al. ${ }^{[6]}$ reviewed 21 patients attending a lymphoma clinic. Of these patients, 13 were HIV-positive and 11 were on antiretroviral (ARV) therapy. They identified 18 (86\%) patients who had undergone failed TB treatment in the 12 months before their histological confirmation of lymphoma. None had a confirmed TB culture before starting treatment. All these patients subjectively reported TB treatment failure, with a median duration of 5 months' treatment. Only 7 (39\%) patients reported being followed-up at 1 month post treatment initiation. Fine-needle aspiration (FNA) was suggested in the work-up of all patients with lymphadenopathy, and lymph node (LN) biopsy for patients failing to respond to empirical TB treatment after 1 month.

It may also be difficult to distinguish TB from relapsed lymphoma. Karakas et al. ${ }^{[7]}$ evaluated the association of Hodgkin's lymphoma (HL) and pulmonary TB, reviewing the medical records of 70 patients. A total of 27 patients (38\%) had mediastinal-pulmonary involvement initially, and systemic symptoms were present in 37 (52\%) patients. Fourteen patients (20\%) had pulmonary TB: 3 had TB before HL, 2 had TB and HL concomitantly at initial diagnosis, 7 had TB during lymphoma therapy and 2 after the cessation of lymphoma treatment. Eleven patients with pulmonary TB had diffuse pulmonary infiltrations and mediastinal enlargement on computed tomography (CT) and X-ray. The radiologic differentiation could not be made on 9 patients who had a thoracic CT scan.

Lymphoma diagnosis depends on morphology, immunohistochemistry and flow cytometry (FC) and, where appropriate, molecular studies to accurately categorise the lymphoma. An FNA is inadequate for initial diagnosis. An incisional or excisional biopsy is preferred to provide adequate tissue for these examinations.

Transbronchial needle aspiration (TBNA) is a minimally invasive bronchoscopic technique that is cost-effective and safe for diagnosing mediastinal and hilar adenopathy in patients with lung cancer, other malignancies, sarcoidosis and infectious processes such as TB. ${ }^{[8,9]}$ Most of the studies on the diagnostic yield of TBNA for mediastinal and hilar adenopathy have been performed in patients with lung cancer. Few 
studies have analysed the sensitivity, specificity and predictive values of TBNA for diagnosing lymphoma. Therefore, it is difficult to compare the results among studies and perform a global evaluation of the technique.

In ill patients unable to undergo a definitive diagnostic procedure, TBNA is safe and has a high diagnostic yield. Ghamande et al. ${ }^{[10]}$ evaluated TBNA in 8 ventilated patients. TBNA showed a sensitivity of $83 \%$, specificity of $100 \%$, positive predictive value (PPV) of $100 \%$, and negative predictive value (NPV) of 50\%. Diagnoses were carcinoma in 4 patients and post-transplantation lymphoproliferative disorder in 1 patient. Of the 3 patients with negative TBNA results, 2 underwent mediastinoscopy (MDS) (non-small-cell carcinoma and inflamed tissue). The 3rd patient could not undergo a MDS because of coagulopathy and no autopsy was performed. TBNA led to management changes in $5(63 \%)$ patients. There were no complications from the TBNAs.

FC has been shown to improve diagnostic yield. In a study of 285 patients, by Schmid et al., ${ }^{[11]}$ cytological specimens were retrospectively analysed and correlated with histology and follow-up. Cytologically diagnosed malignancy was confirmed in all histologically examined cases. In $92 \%$ of reactive cytological cases, a benign process was diagnosed histologically. Correlation with histology showed a sensitivity of $98 \%$ and a specificity of $100 \%$ for cytology.

Another study by Gorczyca et al. ${ }^{[12]}$ reviewed 100 patients presenting with mediastinal lesions. Samples were adequate for FC evaluation in $95 \%$. Results showed that in $97 \%, 67 \%, 78 \%, 88 \%$ and $100 \%$ cases of B-cell lymphoma, T-cell lymphoma, carcinoma, T-cell acute lymphoblastic leukaemia/lymphoblastic lymphoma and thymoma/thymic hyperplasia, respectively, the diagnosis could be reached by FC alone. Excluding HL, the general sensitivity of FC in diagnosing mediastinal tumours was $92 \%$ and no false-positive (FP) results were encountered. Flow methodology has the advantage of rapid turnaround time as well as high sensitivity, enabling patients with large anterior mediastinal masses and/or superior vena cava syndrome to begin treatment promptly.

MDS is currently used extensively for the diagnosis of thoracic disease and staging of malignancies. ${ }^{[13]}$ Positron-emission tomography (PET) scans have recently raised doubts as to the importance of MDS in the diagnosis and staging of lung cancer, with sensitivity and specificity almost approaching that of MDS. ${ }^{[14]}$ However, since histological diagnosis is the issue, especially where a differentiation needs to be made between sarcoidosis, TB and lymphoma, MDS remains the diagnostic procedure of choice. In a study by Nalladaru et al.. ${ }^{[15]}$ MDS resulted in a definitive diagnosis in $97 \%$ of patients with isolated mediastinal lymphadenopathy.

Sonar-guided FNA procedures (endoscopic ultrasound (EUS) and endobronchial ultrasound (EBUS)) are on the rise, especially for the diagnosis of primary and metastatic lesions. This has been increasingly noted for lung, oesophageal and pancreatic carcinomas. Determining a definitive diagnosis of lymphoma by aspiration cytology is particularly important for patients whose condition may be too unstable to undergo general anaesthesia and open surgical biopsy. Earlier studies have demonstrated that EUS-FNA biopsy is a safe and effective procedure without significant complications. It also is less costly than other methods of obtaining tissue for diagnosis, including MDS, thoracotomy and CT-guided biopsies. ${ }^{[16]}$ EUS-FNA allows access to deep-seated LNs and allows sampling of lesions that are small $(<25 \mathrm{~mm})$ and may be difficult to sample using other

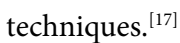

Nunez et al. ${ }^{[18]}$ analysed 1338 cases of EUS and EBUS-FNAB of deep-seated LNs. Results showed that, in association with FC and/ or immunohistochemical analysis, EUS-FNAB was $89 \%$ sensitive and $100 \%$ specific in the diagnosis of primary and recurrent deep-seated non-HL and HL. Currently, the diagnosis of lymphoma is based on the WHO classification system. A study by Yasuda et al. ${ }^{[19]}$ of 152 patients with lymphoma showed that EUS-FNAB was able to determine the subclassification, based on the WHO classification, in $89 \%$ of cases.

\section{Objective}

The purpose of this study was to evaluate the diagnostic yield of TBNA for diagnosing mediastinal and hilar adenopathy from suspected lymphoma in a cohort of patients evaluated at Tygerberg Hospital over a 3-year period (July 2010 - June 2013).

\section{Methods}

\section{Study design and population}

We performed a retrospective analysis of the collected data of patients with thoracic CT-detected mediastinal and hilar adenopathy adjacent to the tracheobronchial tree (with the short axis $\geq 1 \mathrm{~cm}$ ) who underwent TBNA. Approximately 1000 bronchoscopies are performed annually at Tygerberg Hospital. TBNAs are performed by experienced bronchoscopists, and registrars under their supervision. We included all adult patients who underwent bronchoscopy for evaluation of mediastinal or hilar lymph adenopathy secondary to suspected lymphoma. Histology obtained from any method was used as the gold standard. Approval was granted by Stellenbosch University Health Research Ethics Committee (HREC ref.: S13/10/192).

\section{TBNA materials and methods}

The Respiratory Division's bronchoscopy reports were used as source documents. In general, patients underwent conscious sedation with intravenous propofol. Bronchoscopy was performed transnasally/orally using $2 \%$ lignocaine jelly/xylocaine spray as local anaesthesia. TBNA of selected mediastinal or hilar adenopathy stations was performed. The insertion point was determined after an analysis of thoracic CT with or without PET-CT. In most (84\%) cases, aspiration was not ultrasoundguided as the hospital obtained EBUS after the start of the study period; therefore, EBUS was used in only 4 (16\%) patients. A cytopathologist was present during most (92\%) procedures and made an immediate microscopic evaluation (rapid on-site evaluation (ROSE)) of the cytology samples. Suspected lymphoma was defined as cytology compatible with or suggestive of lymphoma. Suspected malignancy included atypical cells, undifferentiated malignant cells or an abnormal cell population.

\section{Data collection interpretation}

Samples with high lymphoid cellularity (suggesting an LN puncture), neoplastic cells or cytological findings that allowed for a specific diagnosis were considered adequate. Samples with too few cells for cytological evaluation or tracheobronchial wall cellularity (suggesting superficial puncture) were considered non-adequate. Clinical data included patient demographics, HIV status, current/previous TB or malignancies, LN involvement, ROSE, cytological diagnosis, FC results, histological diagnosis and procedural complications.

Samples with a cytological diagnosis of lymphoma confirmed by a surgical technique were considered true positives (TPs) and FPs indicated cases with confirmed negative histology. Cases yielding only 
a lymphoid cellularity in patients with specific pathologies such as carcinomas, TB or sarcoidosis on surgical biopsies were considered true negatives (TNs). False-negatives (FNs) indicated cases with negative cytology, but positive histology.

\section{Statistical analysis}

Qualitative variables were reported as absolute frequencies and percentages, and numeric variables as median (range). Sensitivity, specificity, PPV and NPV were determined using the standard definitions.

\section{Results}

Population description

Data from 78 patients with clinical or radiological suspicion of lymphoma were evaluated for the study. All underwent bronchoscopy and TBNA of mediastinal and/or hilar LNs. Patients with histological tissue confirmation of cytological diagnosis were included. The final study population comprised 25 patients. The median age of the patients was $44(18-82)$ years and $16(64 \%)$ were male. Seven (28\%) patients were HIV-positive and of these, 5 (71\%) were on ARV

Table 1. Patient characteristics, TBNA and biopsy results

\begin{tabular}{|c|c|c|c|c|c|c|}
\hline $\begin{array}{l}\text { Known } \\
\text { malignancy } \\
\text { and TB }\end{array}$ & HIV & ROSE & TBNA cytology & $\begin{array}{l}\text { Flow } \\
\text { cytometry }\end{array}$ & TB & Tissue, histology \\
\hline $\mathrm{HL}$ & - & Atypical cells & $\mathrm{HL}$ & & & LN, HL \\
\hline $\mathrm{HL}$ & + & Nil specific & $\begin{array}{l}\text { Reactive lymphocytes } \\
\text { (EBUS) }\end{array}$ & & - & $\mathrm{LN}, \mathrm{HL}$ \\
\hline DLBCL & + & ? Lymphoma & ? DLBCL & & & LN, DLBCL \\
\hline FL & Unknown & Atypical cells & Atypical cells & FL/DLBCL & + & LN, DLBCL \\
\hline CLL & - & CLL & SLL & CLL & & LN, SLL \\
\hline CLL & + & CLL & $\begin{array}{l}\text { Reactive lymphocytes } \\
\text { Abnormal cell } \\
\text { population }\end{array}$ & & - & Arm mass, SLL \\
\hline CLL & - & Nil specific & Reactive lymphocytes & & & Scalp mass, SLL \\
\hline \multirow[t]{5}{*}{ AML } & - & Nil specific & Reactive lymphocytes & & - & Skin macules, necrotising granuloma \\
\hline & - & Atypical cells & ? HL & & - & LN, HL \\
\hline & - & Nil specific & Normal mucosa & & - & LN, HL \\
\hline & - & Nil specific & Mucinous (EBUS) & & - & Mediastinoscopy, HL \\
\hline & + & NSCLC & $\begin{array}{l}\text { Undifferentiated } \\
\text { malignant cells }\end{array}$ & Inconclusive & & LN, primary effusion lymphoma \\
\hline $\begin{array}{l}\text { Previous TB } \\
2010\end{array}$ & + & Lymphoid & Reactive lymphocytes & & - & Spleen, marginal zone lymphoma \\
\hline \multirow{5}{*}{$\begin{array}{l}\text { Empirical TB } \\
\text { treatment }\end{array}$} & - & Lymphocytes & Reactive lymphocytes & & - & LN, squamous Ca metastasis \\
\hline & - & Adeno Ca & Normal mucosa & & & Retroperitoneal, adeno Ca metastasis \\
\hline & - & Reactive LN & $\begin{array}{l}\text { Reactive lymphocytes } \\
\text { (EBUS) }\end{array}$ & & - & Transhepatic, GIST \\
\hline & - & & Granuloma & & - & Endobronchial, granuloma \\
\hline & - & Granuloma & Granuloma & & - & Transbronchial, granuloma \\
\hline \multirow{4}{*}{$\begin{array}{l}\text { Empirical TB } \\
\text { treatment }\end{array}$} & + & Granuloma & Granuloma & Insufficient & - & Endobronchial, granuloma \\
\hline & - & $\begin{array}{l}\text { Necrotising } \\
\text { granuloma }\end{array}$ & Necrotising granuloma & & + & Endobronchial, granuloma \\
\hline & - & $\begin{array}{l}\text { Lymphocytes/ } \\
\text { macrophages }\end{array}$ & Reactive lymphocytes & & - & Mediastinoscopy, granuloma \\
\hline & - & Normal & $\begin{array}{l}\text { Reactive lymphocytes } \\
\text { (EBUS) }\end{array}$ & & - & Endobronchial, granuloma \\
\hline \multirow{2}{*}{$\begin{array}{l}\text { Empirical TB } \\
\text { treatment }\end{array}$} & - & & Too few cells & & - & Mediastinoscopy, necrotising granuloma \\
\hline & Unknown & Myxoid cells & Myxoid & & - & Mediastinoscopy, reactive LN/bronchial cyst \\
\hline $\begin{array}{l}\text { Previous TB } \\
2011\end{array}$ & + & Granuloma & Normal mucosa & & - & Mediastinoscopy, reactive $\mathrm{LN}$ \\
\hline
\end{tabular}


therapy. Their CD4 counts varied from 32 1537 cells $/ \mathrm{mm}^{3}$. The patient characteristics and final diagnoses are presented in Table 1.

All patients had a contrasted CT scan. Eight (32\%) were known to have haematological malignancy: 2 with HL, 1 diffuse large B-cell lymphoma (DLBCL), 1 follicular lymphoma (FL), 3 chronic lymphocytic leukaemia/ small lymphocytic lymphoma (CLL/SLL), 1 acute myeloid leukaemia (AML). Twelve (48\%) patients had lymphoma confirmed on a histological sample: 5 (42\%) HL, 2 (17\%) DLBCL, 3 (25\%) CLL/SLL, 1 (8\%) splenic/ nodal marginal zone lymphoma, 1 (8\%) primary effusion lymphoma.

The smallest axis diameter of the 38 studied LNs was $10 \mathrm{~mm}$ (range $10-52 \mathrm{~mm}$ ); 23 (61\%) were smaller than $20 \mathrm{~mm}$. A mean of 3.6 (range 1 - 9) passes were performed.

\section{TBNA validity and reliability}

Adequate samples for cytological evaluation were obtained from 22 (88\%) patients. Cytological diagnosis could be made for $8(32 \%)$ patients, including suspected lymphoma $(n=4)$ and granuloma $(n=4)$. Samples were considered non-adequate for 3 (12\%) patients (normal mucosa and too few cells). FC was requested for only 4 (16\%). TB cultures were performed for 19 (76\%). Of these $2(11 \%)$ were positive. Histology provided the final diagnosis for all cases, with 12 lymphoma cases confirmed.

ROSE was performed in 23 (92\%). In 17/23 (74\%) cases similar results were obtained to formal cytology. In the lymphoma subgroup, ROSE showed 7/12 (58\%) cells suspicious of malignancy (2 CLL, 3 atypical cells, 1 possible lymphoma, 1 non-small-cell lung cancer) and 5 (42\%) were non-diagnostic (4 nil specific and 1 lymphoid) with sensitivity for suspicion of malignancy $58 \%$ and specificity $100 \%$. All ROSE and cytology samples suggestive of malignancy were confirmed by histology, indicating excellent PPV.

In the lymphoma subgroup, 7/12 (58\%) patients had cytology suspicious of malignancy (HL, CLL/SLL, possible HL, possible DLBCL, atypical cells, undifferentiated malignant cells, abnormal cell population). Only 2 (17\%) had a specific cytological diagnosis (both patients were known to have the same haematological malignancy). Another 2 had cytology results suggestive of lymphoma (1 known with DLBCL and 1 newly diagnosed with $\mathrm{HL}$ ). Cytology was FN for malignancy in
$5 / 12(42 \%)$ of the lymphoma subgroup (3 reactive lymphocytes, 1 normal mucosa, 1 mucinous). TBNA sensitivity for suspecting lymphoma was $33 \%$ and specificity $100 \%$.

In patients with known haematological malignancies, TBNA cytology showed atypical cells in $5(63 \%)$ and $3(37 \%)$ had FN results. Only 2 (25\%) had a definitive diagnosis and $1(13 \%)$ a suggestive diagnosis of lymphoma. In the 5 newly diagnosed lymphoma patients, TBNA cytology showed atypical cells in $2(40 \%)$ and $\mathrm{FN}$ results in 3 (60\%). Only 1 (20\%) showed cytology suggestive of lymphoma.

Of the study population, $8 / 25$ (32\%) had granulomatous inflammation on histology (6 non-necrotising and 2 necrotising). One of these had a positive TB culture. All 4 cases of cytological granulomatous inflammation were confirmed by histology, with TBNA sensitivity of $50 \%$ and specificity of $100 \%$.

FC was performed on 4 (16\%) TBNA samples. The routine immunophenotypic panel was: $\mathrm{CD}_{3}, \mathrm{CD}_{4}, \mathrm{CD}_{5}, \mathrm{CD}_{8}, \mathrm{CD}_{10}, \mathrm{CD}_{19}$, $\mathrm{CD}_{20}, \mathrm{CD}_{22}, \mathrm{CD}_{23}, \mathrm{CD}_{38}, \mathrm{CD}_{45}$, FMC-7, kappa and lambda $\left(\mathrm{CD}_{45}\right.$ gating strategy). One sample was insufficient for analysis. The other samples demonstrated $1 \mathrm{CLL}, 1 \mathrm{FL}$ relapse/transformation into DLBCL and 1 was inconclusive. Sensitivity was $50 \%$ and specificity $100 \%$. From the source population, 19/78 (25\%) had FC performed.

Lymphoma subtyping was as follows: 5 patients had classic HL (1 nodular sclerosis, 1 mixed cellularity, 1 lymphocyte rich, 1 mixed cellularity nodular sclerosis, 1 lymphocyte depleted), 3 patients had CLL/SLL, 2 patients had DLBCL, 1 patient had splenic and nodal marginal zone lymphoma and 1 patient had primary effusion lymphoma.

MDS was performed in 5 (20\%) patients. One patient had HL. The results are shown in Table 2.

Peripheral LN biopsy confirmed 8/12 (67\%) lymphomas, soft-tissue biopsy 2 (17\%) and splenectomy $1(8 \%)$. MDS yielded $1(8 \%)$ undiagnosed HL. Lymphoma could be confirmed in 10 (82\%) with minor surgical procedures (peripheral LN or soft-tissue biopsy) and only 2 (17\%) required major surgical procedures (MDS or laparotomy).

\section{Complications}

Complications related to TBNA occurred in 3 patients: 1 case of mild, self-limited bleeding at the puncture site, 1 patient was too restless to complete the procedure and 1 patient had laryngospasm.

\section{Discussion}

There is a question as to the accuracy of fineneedle aspiration cytology (FNAC) in the diagnosis of lymphomas, as the tumours often contain malignant and reactive elements and the FNAC may only have sampled the reactive regions. The inherent characteristics of lymphoma (i.e. fibrosis in classic HL) may also prevent cytomorphologists from obtaining enough material for several analyses. Visualising LN changes may help with selecting the right aspiration area and overcome problems with partial fibrotic changes in the affected LN. ${ }^{[20]}$

Another disadvantage of FNAC is that it does not provide the cellular architecture required for accurate subtyping of the lymphoma. The sensitivity of all FNA for the diagnosis of lymphoma (published 2004 - 2014) has been reported to range between $25 \%$ and $95 \% .{ }^{[21]}$

As a result of the deficiencies of FNAC, LN excision or core needle biopsy is required and is the recommended second-line diagnostic procedure. All suspicious LNs should be biopsied following FNA, even if FNA is reported normal or demonstrating reactive changes only. ${ }^{[22]}$ Histological confirmation and subtyping of lymphoma remains the standard precondition for chemotherapy. TBNA/EBUS-TBNA cell block samples ${ }^{[23]}$ and EBUS transbronchial needle forceps biopsies $^{[24]}$ may overcome these deficiencies.

\section{Table 2. Results from mediastinoscopy}

\begin{tabular}{ll}
\hline Cytology result & Final diagnosis \\
\hline Mucinous & $\begin{array}{l}\text { Hodgkin's lymphoma (extranodal) lymphocyte depleted/diffuse } \\
\text { fibrosis type }\end{array}$ \\
Reactive lymphocytes & $\begin{array}{l}\text { Non-necrotising granulomatous inflammation and anthracosis } \\
\text { Normal mucosa }\end{array}$ \\
Reactive LN with anthracosis, fibrosis and scarring \\
Too few cells & Reactive LN and bronchial cyst \\
& Necrotising granulomatous inflammation
\end{tabular}


A limited number of study patients had sonar-guided LN aspiration. In the lymphoma subgroup, no tissue suitable for immunohistochemical analysis (cell block or forceps biopsy) was submitted from TBNA samples. There were also a limited number of FC tests requested. This may explain the low sensitivity (33\%) demonstrated by our study.

The only study done in the last 10 years evaluating the diagnostic yield of conventional (blind) TBNA in the diagnosis of lymphoma (using cytology and/or histology needles) was by Fernández-Villar et $a l .^{[25]}$ in 2010, on 15 patients with lymphoma. They also demonstrated TBNA specificity and PPV of $100 \%$, but poor sensitivity (36\%) and NPV (10\%). Other studies evaluating conventional TBNA had a limited number of patients with lymphoma. Sharafkhaneh et al. ${ }^{[26]}$ had 4 patients, Szlubowski et al. ${ }^{[27]} 2$ patients, Selcuk et al. ${ }^{[28]} 1$ patient and Aliyali et al ${ }^{[29]} 2$ patients, with sensitivity ranging from $0 \%-50 \%$. Most studies focused on lung carcinoma, metastatic disease and sarcoidosis.

Most other recent studies looked at EBUS-guided TBNA (developed in 2002). It was traditionally thought that the small samples of mediastinal LNs obtained by EBUS are inadequate for the diagnosis of lymphoma, as treatment regimens for lymphoma are dependent on the specific subtype and histological grade.

Kennedy et al. ${ }^{[30]}$ carried out a retrospective study in 2007 that included 25 patients with mediastinal adenopathy and suspected lymphomas. EBUS-TBNA (in combination with cytology and immunohistochemistry with/without FC) demonstrated sensitivity, specificity, PPV and NPV of $90 \%, 100 \%, 100 \%$ and $93 \%$, respectively.

In 2010, Steinfort et al. ${ }^{[31]}$ reviewed data from 55 patients with suspected lymphoma who underwent EBUS-TBNA (using a 22-gauge needle and preparing a cell block for immunohistochemical analysis) to evaluate mediastinal lymphadenopathy. They showed that EBUSTBNA provided a diagnosis in 16/21 (76\%) of the lymphoma cases, although 4 patients required further surgical biopsy to completely characterise lymphoma subtypes. Sensitivity and specificity for definitive diagnosis of lymphoma were $57 \%$ and $100 \%$, respectively.

Another study published in 2014 showed similar results. Senturk et al. ${ }^{[32]}$ evaluated EBUS-TBNA (with cell block for immunohistochemistry) in 15 patients with lymphoma. A definitive pathological diagnosis and histological typing were achieved in $87 \%$ of patients. Specificity, NPV and diagnostic accuracy were calculated as $100 \%, 97 \%$ and $97 \%$, respectively.

In these studies, though FC was not routinely requested, tissue for immunohistochemical analysis was submitted. This could explain the increased sensitivity for a definitive diagnosis and subtyping of lymphoma, obviating the need for more invasive surgical biopsy.

Recently, a new 22-gauge needle (SonoTip EBUS Pro with stainless steel) was introduced in Japan, showing promising results. ${ }^{[33]}$

The low cost and few complications justify the continued use of conventional TBNA as the initial diagnostic test for mediastinal and hilar adenopathy, especially when other techniques, such as EBUSTBNA, are unavailable. The diagnostic yield of conventional TBNA could be improved by FC or adequate tissue sampling (with cell block preparation) for immunohistochemical analysis.

In our institution, TBNA is a first-line diagnostic procedure for the initial evaluation of mediastinal and/or hilar lymph adenopathy, while FC is not currently used on a routine basis. If lymphoma is suspected, FC of TBNA samples should be encouraged, as it has been shown to have an excellent correlation with histology. In most (84\%) patients, aspiration was not ultrasound guided. Since beginning the study, our hospital has obtained EBUS, and the use of sonar-guided aspiration (+/- biopsy) and improved specimen preparation (including a cell block) should improve TBNA yield for all pathologies.

\section{Study limitations}

Our study was retrospective and included a small number of patients from a single centre. Neither FC nor cell block preparation was routinely used in the evaluation of samples, and EBUS was only acquired towards the end of the study period. With most studies in the last 10 years that evaluate diagnostic yield of TBNA being sonarguided, it is difficult to compare our results. A larger, randomised controlled prospective study comparing conventional TBNA, FC or cell block preparation, EBUS-TBNA and EBUS transbronchial needle forceps biopsy could overcome these limitations.

\section{Conclusion}

In our study, transbronchial FNAC had a low sensitivity, but high rule-in value for the diagnosis of lymphoma in patients presenting with mediastinal or hilar adenopathy. It is an appropriate first-line diagnostic procedure, but further invasive sampling is required for histological confirmation and subtyping of lymphoma, and in cases where TBNA cytology is negative but clinical or radiological suspicion of lymphoma is high. This could be obviated by the routine request of FC, preparing a cell block during TBNA/EBUS-TBNA and performing transbronchial needle forceps biopsy during EBUS-TBNA. The main advantage of TBNA was shown to be in evaluating persistent disease.

It is possible to provide a definitive diagnosis of new or persistent lymphoma using TBNA, but this is not sufficient to exclude new or persistent lymphoma. If ROSE/cytology is suspicious of lymphoma or shows atypical cells, excisional or incisional biopsy should be the immediate second-line diagnostic step.

\section{References}

1. Herbst MC. CANSA Statistics 2010 National Cancer Registry Report. Fact Sheet on the Top Ten Cancers per Population Group, October 2015

2. Floyd K, Glaziouet P, Timimi H, et al. Global Tuberculosis Report 2015, 20th edition. Geneva: World Health Organization, 2015.

3. The South African National Tuberculosis Control Programme. Practical Guidelines. Pretoria: Department of Health, 2004.

4. World Health Organization. Treatment of Tuberculosis Guidelines. Geneva: WHO 2010 .

5. Wilson D, Nachega J, Morroni C, et al. Diagnosing smear-negative tuberculosis using case definitions and treatment response in HIV-infected adults. Int J Tuber Lung Dis 2006;10(1):31-38.

6. Puvaneswaran B, Shoba B. Misdiagnosis of tuberculosis in patients with lymphoma S Afr Med J 2013;103(1):32-33.

7. Karakas Z, Agaoglu L, Taravari B, et al. Pulmonary tuberculosis in children with Hodgkin's lymphoma. Hematol J 2003;4(1):78-81. DOI:10.1038/sj.thj.6200219

8. Dasgupta A, Mehta AC. Transbronchial needle aspiration. An underused diagnostic technique. Clin Chest Med 1999;20:39-51.

9. Wang KP, Metha A, Turner JF. Transbronchial needle aspiration for cytology and histology specimens. In: Flexible Bronchoscopy, 2nd ed. Cambridge: Blackwell Publishing, 2004:117-137.

10. Ghamande S, Rafanan A, Dweik R, Arroliga AC, Mehta AC. Role of transbronchial needle aspiration in patients receiving mechanical ventilation. Chest 2002;122(3):985989. DOI:10.1378/chest.122.3.985

11. Schmid S, Tinguely M, Cione P, Moch H, Bode B. Flow cytometry as an accurate tool to complement fine needle aspiration cytology in the diagnosis of low grade malignant lymphomas. Cytopathology 2010;22(6):397-406. DOI:10.1111/j.13652303.2010.00801.x

12. Gorczyca W, Tugulea S, Liu Z, et al. Flow cytometry in the diagnosis of mediastinal tumors with emphasis on differentiating thymocytes from precursor t-lymphoblastic lymphoma/leukemia. Leuk Lymphoma 2004;45(3):529-538. DOI:10.1080/10428190 310001598008 
13. Carlens E. Mediastinoscopy: A method for inspection and tissue biopsy in the superior mediastinum. Dis Chest 1959;36:343-349.

14. Lee BE, Redwine J, Foster C, et al. Mediastinoscopy might not be necessary inpatients with non-small cell lung cancer with mediastinal lymph nodes having a maximum standardized uptake value of less than 5.3. J Thorac Cardiovasc Surg 2008;135(3):615619. DOI:10.1016/j.jtcvs.2007.09.029

15. Nalladaru ZM, Wessels A. The role of mediastinoscopy for diagnosis of isolated mediastinal lymphadenopathy. Indian J Surg 2011;73(4):284-286. DOI:10.1007/s12262-011-0282-x

16. Catalano MF, Nayar R, Gress F, et al. EUS-guided fine needle aspiration in mediastinal lymphadenopathy of unknown etiology. Gastrointest Endosc 2002;55(7):863-869. DOI:10.1067/mge.2002.124637

17. Jhala NC, Jhala D, Eltoum I, et al. Endoscopic ultrasound-guided fine-needle aspiration biopsy: A powerful tool to obtain samples from small lesions. Cancer 2004;102(4):239-246. DOI:10.1002/cncr.20451

18. Nunez AL, Jhala NC, Carroll AJ, et al. Endoscopic ultrasound and endobronchial ultrasound-guided fine-needle aspiration of deep-seated lymphadenopathy: Analysis of 1338 cases. Cytojournal 2012;9(1):14. DOI:10.4103/1742-6413.95845

19. Yasuda I, Goto N, Tsurumi H, et al. Endoscopic ultrasound-guided fine needle aspiration biopsy for diagnosis of lymphoproliferative disorders: Feasibility of immunohistological, flow cytometric, and cytogenetic assessments. Am J Gastroenterol 2012;107(3):397-404. DOI:10.1038/ajg.2011.350

20. Kuvezdić KG, Aurer I, Ries S. FNA based diagnosis of head and neck nodal lymphoma. Coll Antropol 2010;34(1):7-12.

21. Health Quality Ontario. The Accuracy of Fine-Needle Aspiration Cytology in the Diagnosis of Lymphoma. Toronto: HQO, 2014:1-19.

22. Morris-Stiff G, Cheang P, Key S, et al. Does the surgeon still have a role to play in the diagnosis and management of lymphomas? World J Surg Oncol 2008;6(1):13. DOI:10.1186/1477-7819-6-13

23. Sanz-Santos J, Serra P, Andreo F, et al. Contribution of cell blocks obtained through endobronchial ultrasound-guided transbronchial needle aspiration to the diagnosis of lung cancer. BMC Cancer 2012;12(1):34. DOI:10.1186/1471-2407-12-34
24. Herth FJF, Schuler H, Gompelmann D, et al. Endobronchial ultrasound-guided lymph node biopsy with transbronchial needle forceps: A pilot study. Eur Respir 2012;39(2):373-377. DOI:10.1183/09031936.00033311

25. Fernández-Villar A, Botana M, Leiro V, et al. Validity and reliability of transbronchial needle aspiration for diagnosing mediastinal adenopathies. BMC Pulm Med 2010;10(1):24. DOI:10.1186/1471-2466-10-24

26. Sharafkhaneh A, Baaklini W, Gorin AB, Green L. Yield of transbronchial needle aspiration in diagnosis of mediastinal lesions. Chest 2003;124(6):2131-2135. DOI:10.1378/chest.124.6.2131

27. Szlubowski A, Kuzdzał J, Soja J. Transbronchial needle aspiration as a diagnostic method in lung cancer and non-malignant mediastinal adenopathy. Pneumonol Alergol Pol 2007;75(1):5-12.

28. Selçuk ZT, Firat P. The diagnostic yield of transbronchial needle aspiration in superior vena cava syndrome. Lung Cancer 2003;42(2):183-188. DOI:10.1016/s01695002(03)00293-9

29. Aliyali M, Shafigh E. Diagnostic yield of transbronchial needle aspiration in intrathoracic lymphadenopathy. Tanaffos 2008;7(2):23-27.

30. Kennedy MP, Jimenez CA, Bruzzi JF, et al. Endobronchial ultrasound-guided transbronchial needle aspiration in the diagnosis of lymphoma. Thorax 2008;63(4):360-365.

31. Steinfort DP, Conron M, Tsui A, et al. Endobronchial ultrasound-guided transbronchial needle aspiration for the evaluation of suspected lymphoma. J Thorac Oncol 2010;5(6):804-809. DOI:10.1097/jto.0b013e3181d873be

32. Senturk A, Babaoglu E, Kilic H, et al. Endobronchial ultrasound-guided transbronchial needle aspiration in the diagnosis of lymphoma. Asian Pac J Cancer Prev 2014;15(10):4169-4173.

33. Izumo T, Sasada S, Watanabe J, et al. Comparison of two $22 \mathrm{G}$ aspiration needles for histologic sampling during endobronchial ultrasound-guided transbronchial needle aspiration (EBUS-TBNA). Jpn J Clin Oncol 2014;44(9):841-845. DOI:10.1093/jjco/ hyu095 